\title{
Lower lung function associates with cessation of menstruation: UK Biobank data
}

\author{
André F.S. Amaral ${ }^{1,2}$, David P. Strachan ${ }^{2,3}$, Francisco Gómez Real ${ }^{4,5}$, \\ Peter G.J. Burney ${ }^{1,2}$ and Deborah L. Jarvis ${ }^{1,2}$
}

Affiliations: ${ }^{1}$ Respiratory Epidemiology, Occupational Medicine and Public Health, National Heart and Lung Institute, Imperial College, London, UK. ${ }^{2}$ MRC-PHE Centre for the Environment and Health, London, UK. ${ }^{3}$ Population Health Research Institute, St George's University of London, London, UK. "Dept of Clinical Science, University of Bergen, Bergen, Norway. ${ }^{5}$ Dept of Gynecology and Obstetrics, Haukeland University Hospital, Bergen, Norway.

Correspondence: André F.S. Amaral, Respiratory Epidemiology, Occupational Medicine and Public Health, National Heart and Lung Institute, Imperial College London, Emmanuel Kaye Building, 1B Manresa Road, London SW3 6LR, UK. E-mail: a.amaral囚imperial.ac.uk

ABSTRACT Little is known about the effect of cessation of menstruation on lung function. The aims of the study were to examine the association of lung function with natural and surgical cessation of menstruation, and assess whether lower lung function is associated with earlier age at cessation of menstruation.

The study was performed in 141076 women from the UK Biobank, who had provided acceptable and reproducible spirometry measurements and information on menstrual status. The associations of lung function (forced vital capacity (FVC), forced expiratory volume in $1 \mathrm{~s}$ (FEV1), spirometric restriction (FVC < lower limit of normal (LLN)), airflow obstruction ( $\mathrm{FEV} 1 / \mathrm{FVC}<\mathrm{LLN})$ ) with cessation of menstruation and age at cessation of menstruation were assessed using regression analysis.

Women who had natural cessation of menstruation showed a lower FVC ( $-42 \mathrm{~mL}$; 95\% CI $-53--30)$ and FEV1 (-34 mL; 95\% CI -43- -24) and higher risk of spirometric restriction (adjusted odds ratio 1.27; 95\% CI 1.18-1.37) than women still menstruating. These associations were stronger in women who had had a hysterectomy and/or oophorectomy. The earlier the natural cessation of menstruation, the lower the lung function. There was no clear association of lung function with age at hysterectomy and/or oophorectomy. Airflow obstruction was not associated with cessation of menstruation.

Lower lung function associates with cessation of menstruation, especially if it occurs early in life.

@ERSpublications

Low lung function associates with menopause, especially if it occurs early in life http://ow.ly/RWRt3021tBY

This article has supplementary material available from erj.ersjournals.com

A press release for this article is available from erj.ersjournals.com

Received: Feb 252016 | Accepted after revision: June 262016 | First published online: Sept 222016

Support statement: British Lung Foundation (RHotN12-14). Funding information for this article has been deposited with the Open Funder Registry. The funder of this study had no role in study design, data analysis and interpretation of results, or writing of the manuscript.

Conflict of interest: Disclosures can be found alongside this article at erj.ersjournals.com

Copyright OERS 2016 


\section{Introduction}

In adult males and females, low lung function is a major predictor of mortality, even in nonsmokers and in those without symptoms of lung disease [1,2]. Although many authors have linked this to forced expiratory volume in $1 \mathrm{~s}(\mathrm{FEV} 1)$ and airflow obstruction, more recent work has shown that the strong association is between mortality and forced vital capacity (FVC), rather than airflow obstruction $[1,3]$. Multiple risk factors for low lung function have been put forward, including early life exposures, lifestyle factors (mainly smoking), environmental and occupational exposures, and the presence of asthma at any point during the lifetime [4]. These may operate by causing poor lung development in utero or during childhood, leading to failure to achieve maximal lung function early in life, or through more rapid decline in lung function with age during adulthood.

Differences in lung development and lung function between males and females are present throughout the life course, and have been attributed to sex-specific responses to environmental or lifestyle insults and to the effect of sex hormones $[5,6]$. Menopause, i.e. the cessation of menstruation, usually in response to a natural age-related decline in sex hormones, commonly occurs between 45 and 55 years of age, although there is considerable variation [7,8]. Cessation of menstruation is sometimes precipitated by surgical interventions such as oophorectomy and hysterectomy. In young women, these interventions may prompt the prescription of hormone replacement therapy (HRT).

The cessation of menstruation has been examined in relation to several chronic diseases and health parameters, but little is known about its effect on lung function. Epidemiologic evidence on the association of menopause with lung function is limited, conflicting, inconclusive and based largely on underpowered cross-sectional studies. In two studies with a combined total of less than 3300 women, the European Community Respiratory Health Survey II and the Mater University of Queensland Study of Pregnancy, menopause was associated with both lower FEV1 and FVC $[9,10]$. In a smaller study, the Isparta Menopause and Health Study, menopause was associated with lower FEV1 and FVC, but this was explained by age, and it was not associated with airflow obstruction [11]. In contrast, in another cross-sectional study of just over 3700 women from the Health Survey for England, natural and surgical cessation of menstruation were associated with less airflow obstruction, and natural menopause was associated with higher FEV1, although only in women aged 55-64 years [12].

Most women undergo natural menopause, i.e. natural cessation of menstruation, around the age of 50 years [8], which means that with increasing life expectancy they will spend a large part of their lives in a post-menopausal state. Thus, it is important to expand our understanding of the impact that menopause and other types of cessation of menstruation have on the lung.

The aims of this study were to: 1) examine the association of lung function with natural and surgical cessation of menstruation in the population-based sample of the UK Biobank, and 2) assess whether lower lung function is associated with earlier age at natural menopause.

\section{Methods}

Study participants

The UK Biobank is a very large multicentre population-based study, established to allow the identification and improved understanding of the genetic and nongenetic determinants of disease in middle-aged and elderly adults [13]. The 273467 women who took part in the UK Biobank were assessed, between 2006 and 2010, in 22 centres across England, Wales and Scotland located in areas with sufficient population aged 40-69 years living within $16 \mathrm{~km}$ of the testing centre. During assessment, participants completed a touchscreen questionnaire to provide information on their lifestyle, medical and family history, and underwent physical measurements, including spirometry.

The UK National Research Ethics Service Committee North West (Haydock) approved the study and electronic written consent from each participant was obtained.

\section{Lung function outcomes}

Lung function was assessed, in each centre, by nurses or healthcare technicians trained in spirometry following a standard protocol and using a Vitalograph Pneumotrac 6800 spirometer (Vitalograph, Buckingham, UK). Participants did not perform spirometry if they answered "yes" to or were unsure of the following: chest infection in the last month (i.e. influenza, bronchitis, severe cold, pneumonia); history of detached retina; heart attack or surgery to eyes, chest or abdomen in last 3 months; history of collapsed lung; pregnancy (first or third trimester); and currently on medication for tuberculosis. The aim was to record two acceptable blows from a maximum of three attempts. The spirometer software compared the acceptability of the first two blows and, if acceptable (defined as a $\leqslant 5 \%$ difference in FVC and FEV1), indicated to the fieldworkers that the third blow was not required. Post-bronchodilator measurements were 
not performed. Analyses in this report were conducted using data from women who had provided at least two spirograms complying with the following criteria: 1) without cough; 2) back-extrapolated volume $<5 \%$ FVC (or $<150 \mathrm{~mL}$ if greater); 3) flow $<25 \mathrm{~mL}$ in final $1 \mathrm{~s}$ of forced expiratory time (FET); 4) both FEV 1 and FVC reproducible; and 5) FET $\geqslant 6 \mathrm{~s}$ on best curve (highest FEV1+FVC) (figure 1).

Outcome measures were: 1) FVC; 2) FEV1; 3) spirometric restriction, defined as FVC < lower limit of normal (LLN) for age and height, based on reference equations for Caucasians from the third US National Health and Nutrition Examination Survey (NHANES III) [14]; and 4) airflow obstruction, defined as FEV1/FVC <LLN, based on the same reference population.

\section{Menstrual status}

Menstrual status was considered in six groups (regular menstruation; irregular menstruation; natural menopause; hysterectomy only; oophorectomy only; oophorectomy plus hysterectomy) and their definition was based on a series of questions. Women who replied "no" to the question "Have you had your menopause (periods stopped)?" were also asked "How many days is your usual menstrual cycle?" If they provided a number to this question, they were classified as having "regular menstruation" (median (interquartile range (IQR)) menstrual cycle length was 28 (25-28) days). If they chose the option "irregular cycle" they were classified as having "irregular menstruation".

Women were considered to have had "natural menopause" if they replied "yes" to the question on menopause, and "no" to both "Have you had both ovaries removed?" (oophorectomy) and "Have you had a hysterectomy (womb removed)?" (hysterectomy). Those whose ovaries and/or uterus were removed after their natural cessation of menstruation were classified has having had "natural menopause". Women were

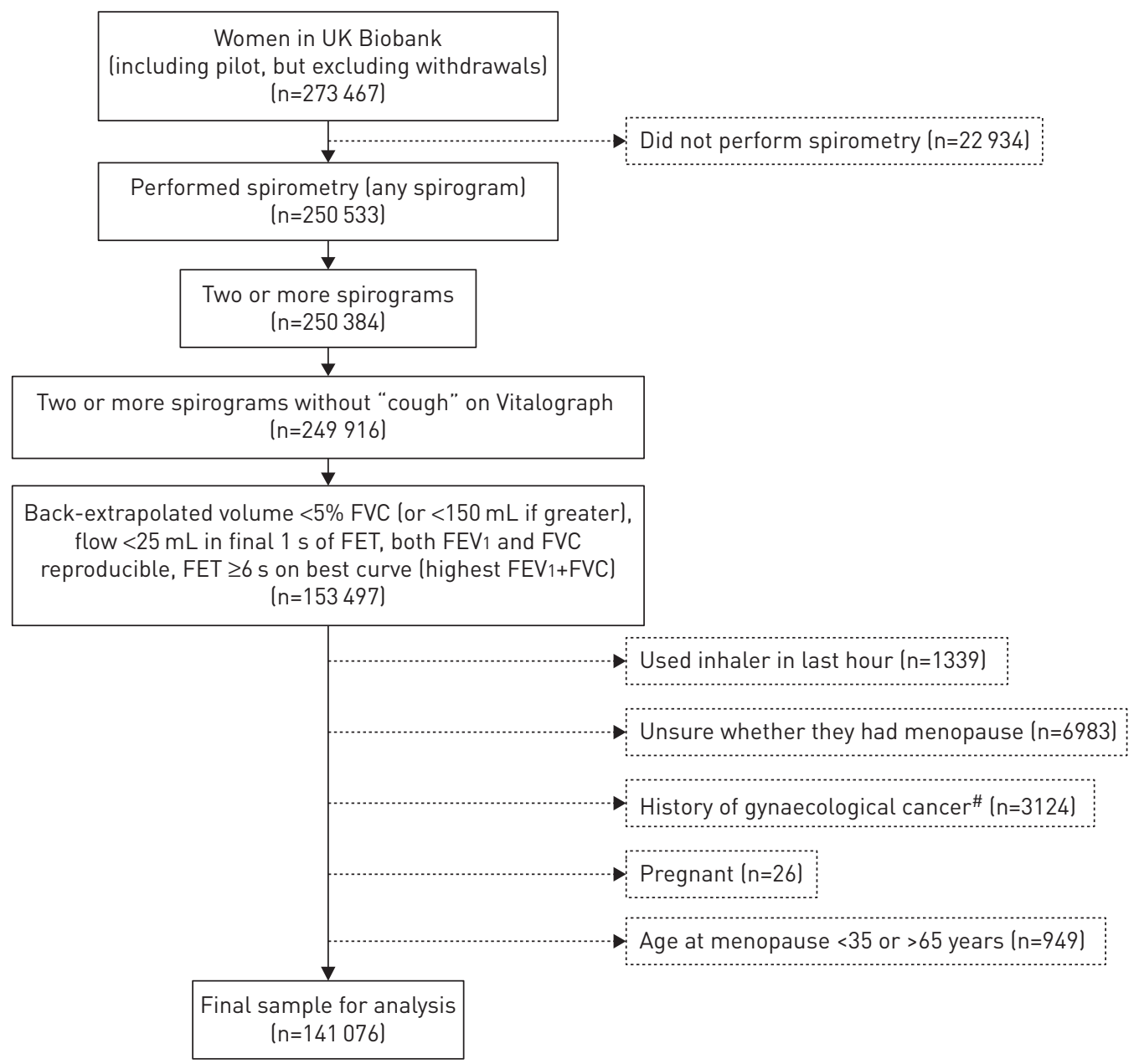

FIGURE 1 Selection of participants in the study. FVC: forced vital capacity; FET: forced expiratory time; FEV1: forced expiratory volume in $1 \mathrm{~s}$. \#: at least one of female genital tract cancer; ovarian cancer; uterine/ endometrial cancer; cervical cancer; vaginal cancer; vulval cancer; cervical intra-epithelial neoplasia/ pre-cancer cells cervix; fallopian tube cancer. 
classified into three other groups depending on the type of surgical cessation of menstruation: "hysterectomy only", "oophorectomy only" and "oophorectomy plus hysterectomy". Women unsure whether they had menopause were excluded from the analysis.

\section{Statistical analysis}

Following exclusions of women who had used an inhaler in the last hour prior to spirometry, women unsure whether they had menopause, those who were pregnant or reported a history of gynaecological cancer and those who had reported an age at menopause $<35$ or $>65$ years, the final sample for analysis comprised 141076 women.

The association of menstrual status with FVC and FEV1 was examined using linear regression. For the association with FVC $<\mathrm{LLN}$ and $\mathrm{FEV} 1 / \mathrm{FVC}<\mathrm{LLN}$, logistic regression was used instead. Three models adjusting for potential confounders were used: Model 1, adjusted for recruitment centre, age (restricted cubic splines with five knots), ethnicity (Caucasian, non-Caucasian), standing height (continuous) and Townsend deprivation index (continuous); Model 2, further adjusted for reproductive characteristics (age at menarche (continuous), oral contraceptive ever use (yes, no), hormone replacement therapy use (never, ex-user, current user) and number of live births $(\mathrm{n}=0,1,2,3,4, \geqslant 5))$; and Model 3, further adjusted for lifestyle-related characteristics (smoking status (lifetime nonsmoker, ex-smoker, current smoker), pack-years (continuous) and body mass index (BMI; <18.5, 18.5-24.99, 25.00-29.99 and $\left.>29.99 \mathrm{~kg} \cdot \mathrm{m}^{-2}\right)$ ). In a sensitivity analysis, the associations of lung function with menstrual status were re-examined excluding women: 1) of non-Caucasian ethnicity; 2) with self-reported doctor-diagnosed asthma and/or chronic obstructive pulmonary disease (COPD); 3) who ever used HRT; 4) who ever smoked; and 5) those with an extreme BMI $\left(<18.5\right.$ or $\left.>29.99 \mathrm{~kg} \cdot \mathrm{m}^{-2}\right)$.

The association of lung function with natural menopause was further assessed by age group (age quintiles among women who had ceased menstruating) and according to age in years at menopause $(<45(10 \%)$, $45-47(15 \%), 48-53(50 \%), 54-55(15 \%)$ and $\geqslant 56(10 \%))$, with natural menopause between the age of 48 and 53 years as reference. In order to examine whether surgical cessation of menstruation is worse than natural cessation, similar analyses were carried out for hysterectomy only and oophorectomy plus hysterectomy among women who have never taken HRT. These analyses were not carried out for women who had had an oophorectomy only due to the small size of this group $(n=214)$.

Results were considered statistically significant when $\mathrm{p}<0.05$. Statistical analysis was performed using Stata version 14 (StataCorp, College Station, TX, USA).

\section{Results}

Characteristics of the 141076 women included in this study are shown in table 1. As compared with women with regular or irregular menstruation, women who had stopped menstruating were older, more likely to be ex-smokers and less likely to have ever used a contraceptive pill. More than two-thirds of the women who had had surgical cessation of menstruation were ex- or current users of HRT. Ever-users of HRT were more likely to be ever-smokers and heavier than those who have never taken HRT (online supplementary table S1).

The prevalence of spirometric restriction was higher in women who had stopped menstruating, while the prevalence of airflow obstruction was slightly higher in women still menstruating. Both FEV1 and FVC were lower in women who had stopped menstruating.

Just over half of the women who had natural menopause reported their last menstruation to have occurred at an age between 48 and 53 years. Most women who had undergone surgical cessation of menstruation had their surgical intervention before they were 48 years of age (online supplementary figure S1).

Women excluded from the analyses due to low quality of spirometric data were similar to those included (online supplementary table S2).

\section{Association of lung function with natural cessation of menstruation}

After full adjustment (Model 3), women with irregular menstruation and natural menopause showed lower FVC (irregular menstruation: $-17 \mathrm{~mL}$ (95\% CI -31--3); natural menopause: $-42 \mathrm{~mL}$ (95\% CI -53--30)), lower FEV1 (irregular menstruation: $-12 \mathrm{~mL}$ (95\% CI -24-0); natural menopause: $-34 \mathrm{~mL}$ (95\% CI $-43--24)$ ) and higher risk of spirometric restriction (irregular menstruation: odds ratio (OR) 1.16 (95\% CI 1.05-1.28); natural menopause: OR 1.27 (95\% CI 1.18-1.37)) than those still menstruating. There was no association of airflow obstruction with irregular menstruation or natural menopause (table 2).

Sensitivity analyses restricted to 33406 Caucasian women who had never smoked, had a BMI of 18.5-29.99 kg.m ${ }^{-2}$, did not have doctor-diagnosed asthma or COPD and had never used HRT did not materially alter the results reported above (table 2 and online supplementary table S4). 
TABLE 1 Characteristics of women in the UK Biobank included in this study

\begin{tabular}{|c|c|c|c|c|c|c|c|}
\hline & \multirow{2}{*}{$\begin{array}{c}\text { Regular } \\
\text { menstruation }\end{array}$} & \multirow{2}{*}{$\begin{array}{l}\text { Irregular } \\
\text { menstruation }\end{array}$} & \multirow{2}{*}{$\begin{array}{c}\text { Natural } \\
\text { menopause }\end{array}$} & \multicolumn{3}{|c|}{ Surgical intervention before menopause } & \multirow[t]{2}{*}{ Total population } \\
\hline & & & & $\begin{array}{l}\text { Hysterectomy } \\
\text { only }\end{array}$ & $\begin{array}{l}\text { Oophorectomy } \\
\text { only }\end{array}$ & $\begin{array}{l}\text { Oophorectomy plus } \\
\text { hysterectomy }\end{array}$ & \\
\hline Subjects $\mathrm{n}$ & 29249 & 6058 & 83023 & 12551 & 214 & 9981 & 141076 \\
\hline Age years & $45(43-49)$ & $48(45-51)$ & $60(56-64)$ & $61(55-64)$ & $57(51-63)$ & $61(55-64)$ & $58(50-63)$ \\
\hline Age at menarche years" & $13(12-14)$ & $13(12-14)$ & $13(12-14)$ & $13(12-14)$ & $13(12-14)$ & $13(12-14)$ & $13(12-14)$ \\
\hline Age at cessation of menstruation years ${ }^{\pi}$ & & & $51(48-53)$ & $41(38-45)$ & $44(37-50)$ & $46(41-50)$ & \\
\hline \multicolumn{8}{|l|}{ Ethnicity } \\
\hline Caucasian & 92.4 & 95.3 & 96.3 & 96.0 & 92.1 & 95.6 & 95.8 \\
\hline Non-Caucasian & 7.6 & 4.7 & 3.7 & 4.0 & 7.9 & 4.4 & 4.2 \\
\hline Standing height $\mathrm{cm}^{+}$ & $164(160-168)$ & $164(160-168)$ & $162(158-166)$ & $162(158-166)$ & $161(158-166)$ & $162(157-166)$ & $162(158-167)$ \\
\hline $\mathrm{BMI} \mathbf{k g} \cdot \mathrm{m}^{-2 \S}$ & $25.3(22.8-28.8)$ & $25.7(23.0-29.6)$ & $26.0(23.5-29.3)$ & $27.1(24.4-30.7)$ & $26.0(23.7-30.3)$ & $27.2(24.3-30.9)$ & $26.0(23.4-29.5)$ \\
\hline $\begin{array}{l}\text { Oral contraceptive lever) use }{ }^{f} \\
\text { Hormone replacement therapy }\end{array}$ & 89.2 & 92.2 & 79.3 & 80.3 & 78.9 & 78.9 & 82.0 \\
\hline Never used & 96.7 & 95.5 & 55.3 & 39.7 & 51.6 & 14.8 & 61.3 \\
\hline Ex-user & 1.9 & 3.2 & 39.6 & 49.2 & 40.4 & 64.6 & 32.9 \\
\hline Current user & 1.4 & 1.3 & 5.1 & 11.1 & 8.0 & 20.6 & 5.8 \\
\hline \multicolumn{8}{|l|}{ Live births } \\
\hline 0 & 25.4 & 25.3 & 16.2 & 10.4 & 22.0 & 15.4 & 17.9 \\
\hline $1-2$ & 55.1 & 55.9 & 58.8 & 59.3 & 49.5 & 58.0 & 58.0 \\
\hline $3-4$ & 18.4 & 17.7 & 23.5 & 28.2 & 26.2 & 24.9 & 22.7 \\
\hline$\geqslant 5$ & 1.1 & 1.1 & 1.5 & 2.1 & 2.3 & 1.7 & 1.4 \\
\hline \multicolumn{8}{|l|}{ Smoking status $^{++}$} \\
\hline Lifetime nonsmoker & 64.5 & 64.7 & 58.2 & 57.8 & 55.4 & 57.3 & 59.7 \\
\hline Ex-smoker & 25.4 & 25.6 & 33.9 & 33.0 & 34.6 & 33.3 & 31.6 \\
\hline Current smoker & 10.1 & 9.7 & 7.9 & 9.2 & 10.0 & 9.4 & 8.7 \\
\hline Pack-years ${ }^{\S \S}$ & $12.8(6.8-21.0)$ & $13.0(6.5-22.0)$ & $18.0(9.0-30.0)$ & $19.0(10.0-30.8)$ & $22.0(10.5-30.0)$ & $19.5(10.0-31.7)$ & $16.5(8.5-28.0)$ \\
\hline Townsend deprivation index ff & $-1.9(-3.5-0.8)$ & $-2.2(-3.6-0.4)$ & $-2.3(-3.7-0.0)$ & $-2.3(-3.7-0.2)$ & $-2.1(-3.7-0.7)$ & $-2.3(-3.7-0.3)$ & $-2.2(-3.7-0.2)$ \\
\hline FVC L & $3.51(3.14-3.91)$ & $3.45(3.09-3.84)$ & $3.02(2.66-3.40)$ & $3.01(2.64-3.40)$ & $3.02(2.60-3.44)$ & $2.98(2.62-3.37)$ & $3.13(2.74-3.55)$ \\
\hline FVC $\%$ pred & $96.1(87.6-104.7)$ & $95.6(86.6-104.2)$ & 93.5 (84.2-102.7) & $92.7(83.5-102.0)$ & $91.8(81.1-101.6)$ & $92.5(83.0-101.7)$ & $94.0(84.8-103.1)$ \\
\hline FEV 1 L & $2.75(2.44-3.05)$ & $2.70(2.40-3.00)$ & $2.30(2.00-2.61)$ & $2.30(2.00-2.61)$ & $2.32(1.97-2.67)$ & $2.28(1.98-2.59)$ & $2.40(2.08-2.74)$ \\
\hline FEV1 \% pred & $93.9(84.9-102.6)$ & $93.6(84.5-102.4)$ & $92.2(81.9-101.9)$ & 91.7 (81.6-101.5) & $92.1(79.5-101.8)$ & $91.4(81.2-101.3)$ & $92.5(82.5-102.0)$ \\
\hline FVC $<$ LLN & 12.3 & 13.3 & 15.5 & 16.9 & 22.4 & 17.6 & 15.0 \\
\hline $\mathrm{FEV}_{1} / \mathrm{FVC}<\mathrm{LLN}$ & 8.9 & 8.0 & 8.7 & 7.4 & 4.7 & 7.9 & 8.6 \\
\hline Doctor-diagnosed asthma & 13.1 & 13.6 & 11.4 & 14.5 & 13.6 & 13.9 & 12.3 \\
\hline Wheeze in last year & 16.6 & 16.7 & 17.1 & 22.2 & 24.6 & 21.6 & 17.8 \\
\hline Doctor-diagnosed COPD & 0.03 & 0.02 & 0.31 & 0.49 & 0.47 & 0.30 & 0.26 \\
\hline
\end{tabular}

Data are presented as median (interquartile range) or \%, unless otherwise stated. BMI: body mass index; FVC: forced vital capacity; FEV1: forced expiratory volume in $1 \mathrm{~s}$; LLN: lower limit of normal. COPD. chronic obstructive pulmonary disease. \#. 3898 missing (regular: 832; irregular: 230; natural: 2276; hysterectomy only: 277; 00phorectomy only: 9; 0ophorectomy plus hysterectomy: 274). ๆ: 5258 missing (natural: 4686; hysterectomy only: 297; oophorectomy only: 27; oophorectomy plus hysterectomy: 248). ${ }^{+}: 81$ missing (regular: 12; irregular: 1; natural: 47; hysterectomy only: 8; oophorectomy plus hysterectomy: 13). §; 1765 missing (regular: 273; irregular: 75; natural: 1065: hysterectomy only: 189; oophorectomy only: 6; oophorectomy plus hysterectomy: 157). ${ }^{f}$ : 289 missing (regular: 62; irregular: 9; natural: 154; hysterectomy only: 30; oophorectomy only: 1; oophorectomy plus hysterectomy: 33). \#\#: 303 missing (regular: 77; irregular: 16; natural: 159; hysterectomy only: 27; oophorectomy only: 1; oophorectomy plus hysterectomy: 23). 171: 74 missing (regular: 13; irregular: 1; natural: 44; hysterectomy only: 10; oophorectomy plus hysterectomy: 6). ${ }^{++}$: 2611 missing (regular: 435; irregular: 101; natural: 1605; hysterectomy only: 263; oophorectomy only: 3; oophorectomy plus hysterectomy: 204). ${ }^{\S} \$ 17129$ missing (natural: 3576 ; irregular: 707 ; natural: 10153 ; hysterectomy only: 1514 ; oophorectomy only: 29 ; oophorectomy plus hysterectomy: 1150). ff: 163 missing (regular: 49; irregular: 9; natural: 80; hysterectomy only: 15; oophorectomy plus hysterectomy: 10). 
TABLE 2 Association of lung function with menstrual status

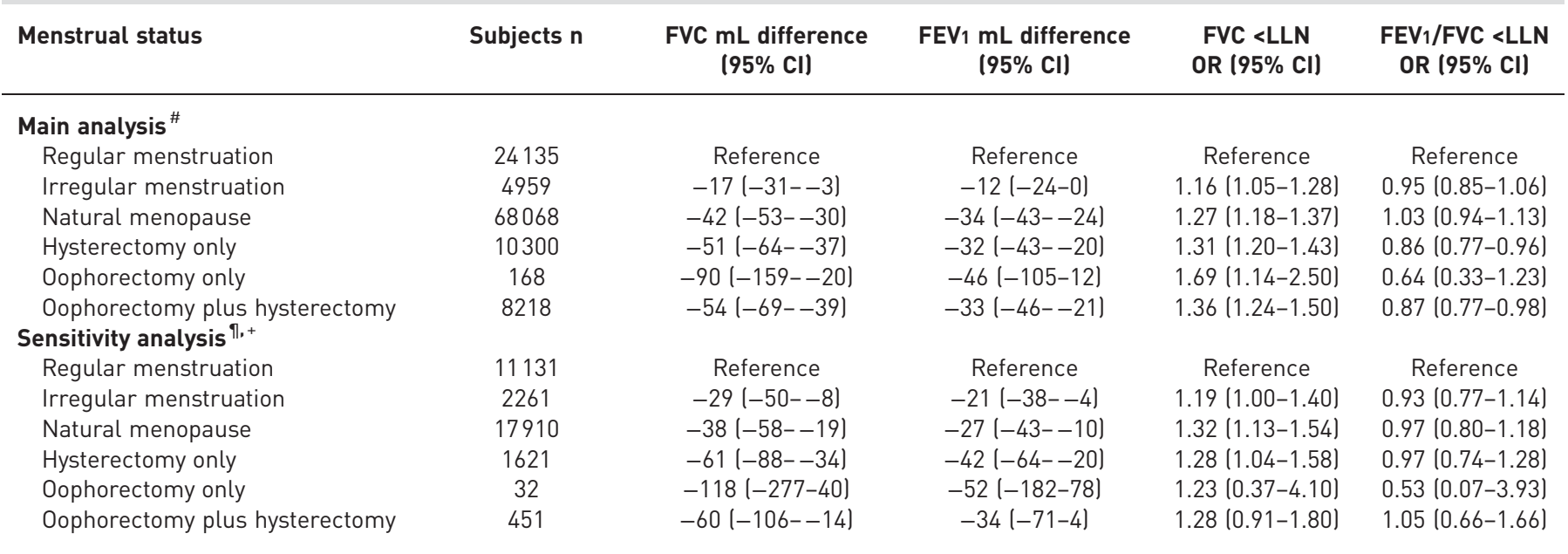

Model 3: Adjusted for centre, age, ethnicity, standing height, Townsend deprivation index, age at menarche, oral contraceptive use, hormone replacement therapy use, number of live births, smoking status, pack-years and body mass index. FVC: forced vital capacity; FEV1: forced expiratory volume in $1 \mathrm{~s}$; LLN: lower limit of normal. " $: \mathrm{n}=115848$; ${ }^{\mathrm{T}}$ : $\mathrm{n}=33406$; ${ }^{+}$: among Caucasian women, who have never smoked, have a body mass index of $18.5-29.99 \mathrm{~kg} \cdot \mathrm{m}^{-2}$, do not have doctor-diagnosed asthma or chronic obstructive pulmonary disease and have never used hormone replacement therapy.

Association of lung function with surgical cessation of menstruation

Women who had undergone hysterectomy, oophorectomy or both had lower FVC (hysterectomy only: $-51 \mathrm{~mL}$ (95\% CI -64--37); oophorectomy only: $-90 \mathrm{~mL}$ (95\% CI -159--20); oophorectomy plus hysterectomy: $-54 \mathrm{~mL}(95 \% \mathrm{CI}-69--39)$ ), lower FEV1 (hysterectomy only: $-32 \mathrm{~mL}$ (95\% CI -43--20); oophorectomy only: $-46 \mathrm{~mL}$ (95\% CI -105-12); oophorectomy plus hysterectomy: $-33 \mathrm{~mL}(95 \% \mathrm{CI}-46--21)$ ) and higher risk of spirometric restriction (hysterectomy only: OR 1.31 (95\% CI 1.20-1.43), oophorectomy only: OR 1.69 (95\% CI 1.14-2.50); oophorectomy plus hysterectomy: OR=1.36 (95\% CI 1.24-1.50)) than women who were menstruating regularly. These associations were present even after sensitivity analyses. Although there was some evidence that women who had hysterectomy with or without oophorectomy showed a lower risk of airflow obstruction, this observation was not seen after sensitivity analyses (table 2 and online supplementary table S4).

Effect of age at natural menopause and surgical cessation of menstruation on lung function Among post-menopausal women, the earlier the natural menopause, the lower the FVC (and FEV1) and higher the risk of spirometric restriction (figures 2 and 3, and online supplementary tables S5-S7). There was no clear association of airflow obstruction with age at natural menopause (figure 4 and online supplementary table S8) nor of lung function with age at surgical cessation of menstruation, i.e. hysterectomy only or oophorectomy plus hysterectomy (online supplementary tables S9 and S10).

\section{Discussion}

In this population-based study of adult females, lower FVC and an increased risk of spirometric restriction were associated with natural and surgical cessation of menstruation, as compared with regular menstruation. The associations with natural menopause were stronger among women who ceased menstruating at an earlier age. These findings were unlikely to be confounded by smoking as they were observed in women who were lifetime nonsmokers.

The strengths of our study are: 1) a very large sample size, which allows for more precise effect estimates; 2) the use of a standardised questionnaire for the collection of data and protocol for spirometry across sites; 3) the use of spirometric measurements with the best quality only; and 4) the adjustment of models for a large set of confounders, including reproductive and lifestyle-related characteristics.

Our study also has limitations. As with all cross-sectional studies, we are unable to draw firm conclusions in terms of temporality. However, we think it is unlikely that low lung function leads to menopause. We are aware that women who smoke tend to have earlier menopause [15] and also low lung function, but as the study is of considerable size, we were able to restrict analyses to a large group of women who have never smoked and see that our findings were unaltered. Both lung function later in life and age at menopause have been associated with socioeconomic status (SES) in childhood [16, 17], but we do not have this SES information. We have adjusted for the Townsend deprivation index of place of residence at 
Natural menopause at $<45$ years

Natural menopause at $45-47$ years

Natural menopause at $48-53$ years

Natural menopause at $54-55$ years

\section{6-59 years $(n=8715)$}

Natural menopause at $<45$ years

Natural menopause at $45-47$ years

Natural menopause at $48-53$ years

Natural menopause at $54-55$ years

Natural menopause at $\geqslant 56$ years

\section{$60-62$ years $(n=6900)$}

Natural menopause at $<45$ years

Natural menopause at $45-47$ years

Natural menopause at $48-53$ years

Natural menopause at $54-55$ years

Natural menopause at $\geqslant 56$ years

\section{3-65 years $(n=5058)$}

Natural menopause at $<45$ years

Natural menopause at $45-47$ years

Natural menopause at $48-53$ years

Natural menopause at $54-55$ years

Natural menopause at $\geqslant 56$ years

\section{6-70 years $(n=5247)$}

Natural menopause at $<45$ years

Natural menopause at $45-47$ years

Natural menopause at $48-53$ years

Natural menopause at $54-55$ years

Natural menopause at $\geqslant 56$ years

$-150-100$

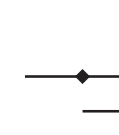

$p_{\text {trend }}$

$4.5 \times 10^{-6}$

0.004

0.3

0.006

$5.7 \times 10^{-4}$

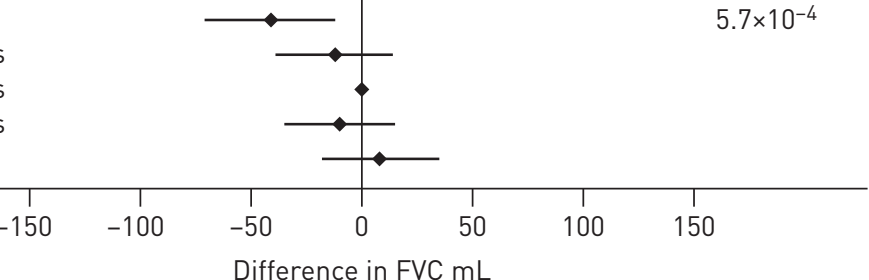

FIGURE 2 Association of forced vital capacity (FVC) with age at natural menopause, by age group. Reference group is women who had menopause at 48-53 years.

the time of assessment. Some women were unsure whether they were menopausal or not and they were excluded from all analyses. It is difficult to predict how the inclusion of these women would affect our results, but since they were likely peri-menopausal (median (IQR) age 51 (48-53) years), and their median FVC $(3.31 \mathrm{~L})$ and FEV1 $(2.57 \mathrm{~L})$ were between those of women with irregular menstruation (FVC $3.45 \mathrm{~L}$; FEV1 $2.70 \mathrm{~L}$ ) and women who had undergone natural menopause (FVC $3.02 \mathrm{~L} ; \mathrm{FEV} 12.30 \mathrm{~L}$ ), it is unlikely that our findings would be different. Those who were included might have responded incorrectly regarding their menstrual status, but this is likely a nondifferential misclassification error leading to underestimation of true effects rather than biased estimates. It is possible that women with worse lung function, and early menopause, died younger, meaning that the associations between menopause and lung function in the older groups may be diluted even further. Endometriosis, an indication for oophorectomy without hysterectomy in young women, has been associated with asthma, but this condition was rare $(<2.5 \%)$ in women who had both ovaries removed. At the assessment centres, spirometry testing did not follow the American Thoracic Society/European Respiratory Society recommendation of performing at least three manoeuvres to measure FVC and FEV1, but we have used only spirometric data that fulfilled all other between- and within-manoeuvre criteria [18]. As we used pre-bronchodilator instead of post-bronchodilator spirometry, we were unable to distinguish COPD from asthma based on lung function. However, the association of airflow obstruction with cessation of menstruation was null. Some could argue that our results may be dependent on the reference equations we used (NHANES III), but we obtained very similar results using the Global Lung Function Initiative equations [19] (data not shown).

To date, this is by far the largest population-based study to assess the association between lung function and menstrual status. The inverse association of FVC and FEV1 with natural menopause is consistent with two previous reports. REAL et al. [9] studied 1274 women from Europe and the USA, and reported much lower 
40-55 years $(n=10994)$

Natural menopause at $<45$ years Natural menopause at $45-47$ years Natural menopause at $48-53$ years Natural menopause at $54-55$ years

\section{6-59 years $(n=8715)$}

Natural menopause at $<45$ years Natural menopause at $45-47$ years Natural menopause at $48-53$ years Natural menopause at $54-55$ years Natural menopause at $\geqslant 56$ years

\section{0-62 years $(n=6900)$}

Natural menopause at $<45$ years Natural menopause at $45-47$ years Natural menopause at $48-53$ years Natural menopause at $54-55$ years Natural menopause at $\geqslant 56$ years

\section{3-65 years $(n=5058)$}

Natural menopause at $<45$ years Natural menopause at $45-47$ years Natural menopause at $48-53$ years Natural menopause at $54-55$ years Natural menopause at $\geqslant 56$ years

\section{6-70 years $(n=5247)$}

Natural menopause at $<45$ years Natural menopause at $45-47$ years Natural menopause at 48-53 years Natural menopause at $54-55$ years Natural menopause at $\geqslant 56$ years $p_{\text {trend }}$

0.1

$5.4 \times 10^{-4}$

0.8

0.05

\begin{tabular}{cc|c|c}
\hline & 1 & 1 & 1 \\
2 & 1 & \\
& 0.5 & Odds ratio of FVC <LLN &
\end{tabular}

FIGURE 3 Association of spirometric restriction (forced vital capacity (FVC) < lower limit of normal (LLN)) with age at natural menopause, by age group. Reference group is women who had menopause at 48-53 years.

FVC and FEV1 in women who had stopped menstruating for at least 6 months, as compared with women with regular menstruation. In an Australian study with 2020 women, HAYATBAKHSH et al. [10] reported lower FVC and FEV1 in post-menopausal women as opposed to pre-menopausal women. These studies reported much larger differences in FVC (REAL et al. [9]: -115 mL; HAYATBAKHSH et al. [10]: -60 mL) and FEV1 (REAL et al. [9]: $-120 \mathrm{~mL}$; НАYатвAKHSH et al. [10]: $-80 \mathrm{~mL}$ ), but the studies were smaller and their analyses were adjusted for considerably less confounders. In contrast, SONGÜR et al. [11] studied 1070 Turkish women and reported no difference in FVC or FEV1 between pre- and post-menopausal women. As with REAL et al. [9], they also showed no association of menopause with airflow obstruction. In an English study with 3724 women, JARVIS and LEYNAERT [12] reported less airflow obstruction in women who had either natural or surgical cessation of menstruation, as compared with menstruating women, but they only observed this among those aged 55-64 years. We observed less airflow obstruction in women who had undergone surgical cessation of menstruation, but this may have been due to use of HRT since exclusion of women who had used this therapy made the association disappear. This finding is supported by at least a population-based observational study and two trials showing better lung function (FVC and FEV1) in post-menopausal women taking HRT [20-22]. None of these studies assessed the association of cessation of menstruation with spirometric restriction. Previous studies have also not examined the effect of different ages at natural menopause, or surgical cessation of menstruation, on lung function.

The biological mechanisms underlying the association of a lower lung function with cessation of menstruation are not fully understood, but may be related to changes in the levels of circulating sex hormones and increased insulin resistance [23]. It has been speculated that with cessation of menstruation the female body may reduce its capacity to detoxify, potentiating the bioaccumulation of chemicals, such as those found in cigarette smoke [24], to levels that could have adverse effects on the lungs. Accelerated bone mass loss [25] and weakened ligaments, which commonly follow the cessation of menstruation, 


\section{0-55 years ( $n=10994$ )}

Natural menopause at $<45$ years

Natural menopause at $45-47$ years

Natural menopause at $48-53$ years

Natural menopause at $54-55$ years

\section{6-59 years $(n=8715)$}

Natural menopause at $<45$ years Natural menopause at $45-47$ years Natural menopause at $48-53$ years Natural menopause at $54-55$ years Natural menopause at $\geqslant 56$ years

\section{0-62 years $(\mathrm{n}=6900)$}

Natural menopause at $<45$ years Natural menopause at 45-47 years Natural menopause at $48-53$ years Natural menopause at $54-55$ years Natural menopause at $\geqslant 56$ years

\section{3-65 years $(n=5058)$}

Natural menopause at $<45$ years Natural menopause at $45-47$ years Natural menopause at $48-53$ years Natural menopause at $54-55$ years Natural menopause at $\geqslant 56$ years

\section{6-70 years $(n=5247)$}

Natural menopause at $<45$ years Natural menopause at $45-47$ years Natural menopause at $48-53$ years Natural menopause at $54-55$ years Natural menopause at $\geqslant 56$ years $p_{\text {trend }}$

Odds ratio of $\mathrm{FEV}_{1} / \mathrm{FVC}<\mathrm{LLN}$

FIGURE 4 Association of airflow obstruction (forced expiratory volume in $1 \mathrm{~s}$ (FEV 1 )/forced vital capacity $(F V C)<$ lower limit of normal (LLN)) with age at natural menopause, by age group. Reference group is women who had menopause at 48-53 years.

could eventually lead to compression and/or fracture of the vertebrae [26] and/or slippage of the ribs, constricting the lungs and reducing their volume, hence increasing the risk of a restrictive lung condition. Medical interventions to treat osteoporotic vertebral compression fractures have been shown to lead to an increase in FVC [27, 28]. Another explanation for worse lung function in women who had an early cessation of menstruation may be related "biological aging". Some studies have shown lower FVC and FEV1 in those with shorter leukocyte telomeres (a marker of faster biological aging) [29, 30], which in turn have been suggested to be a marker of early menopause [31].

In summary, lower lung function, particularly lower FVC, associates with cessation of menstruation. Although more research on this topic is needed and longitudinal studies would be welcome, our findings, together with evidence that low lung function and an early age at menopause have both been associated with increased mortality [3,32], should alert clinicians to the risk of poor lung function in post-menopausal women, especially in those who have an early cessation of menstruation.

\section{Acknowledgements}

This research has been conducted using the UK Biobank Resource. We thank the participants, field workers and data managers of the UK Biobank for their time and cooperation. We also thank James Potts (Imperial College, London, UK) for the local data management at Imperial College.

\section{References}

1 Kannel WB, Hubert H, Lew EA. Vital capacity as a predictor of cardiovascular-disease - the Framingham Study. Am Heart J 1983; 105: 311-315.

2 Schünemann HJ, Dorn J, Grant BJB, et al. Pulmonary function is a long-term predictor of mortality in the general population - 29-year follow-up of the Buffalo Health Study. Chest 2000; 118: 656-664. 
3 Burney PG, Hooper R. Forced vital capacity, airway obstruction and survival in a general population sample from the USA. Thorax 2011; 66: 49-54.

4 Burney P, Jarvis D, Perez-Padilla R. The global burden of chronic respiratory disease in adults. Int J Tuberc Lung Dis 2015; 19: 10-20.

5 Pinkerton KE, Harbaugh M, Han MK, et al. Women and lung disease. Sex differences and global health disparities. Am J Respir Crit Care Med 2015; 192: 11-16.

6 Townsend EA, Miller VM, Prakash YS. Sex differences and sex steroids in lung health and disease. Endocr Rev 2012; 33: 1-47.

7 World Health Organization. Research on the Menopause in the 1990s. Report of a WHO Scientific Group. Geneva, WHO, 1996.

8 Morabia A, Costanza MC. International variability in ages at menarche, first livebirth, and menopause. World Health Organization Collaborative Study of Neoplasia and Steroid Contraceptives. Am J Epidemiol 1998; 148: 1195-1205.

9 Real FG, Svanes C, Omenaas ER, et al. Lung function, respiratory symptoms, and the menopausal transition. J Allergy Clin Immunol 2008; 121: 72-80.

10 Hayatbakhsh MR, Najman JM, O'Callaghan MJ, et al. Association between smoking and respiratory function before and after menopause. Lung 2011; 189: 65-71.

11 Songür N, Aydin ZD, Oztürk O, et al. Respiratory symptoms, pulmonary function, and reproductive history: Isparta Menopause and Health Study. J Womens Health (Larchmt) 2010; 19: 1145-1154.

12 Jarvis D, Leynaert B. The association of asthma, atopy and lung function with hormone replacement therapy and surgical cessation of menstruation in a population-based sample of English women. Allergy 2008; 63: 95-102.

13 UK Biobank Coordinating Centre. UK Biobank: Protocol for a Large-scale Prospective Epidemiological Resource. Stockport, UK Biobank, 2007.

14 Hankinson JL, Odencrantz JR, Fedan KB. Spirometric reference values from a sample of the general U.S. population. Am J Respir Crit Care Med 1999; 159: 179-187.

15 Sun L, Tan L, Yang F, et al. Meta-analysis suggests that smoking is associated with an increased risk of early natural menopause. Menopause 2012; 19: 126-132.

16 Lawlor DA, Ebrahim S, Smith GD. Association between self-reported childhood socioeconomic position and adult lung function: findings from the British Women's Heart and Health Study. Thorax 2004; 59: 199-203.

17 Lawlor DA, Ebrahim S, Smith GD. The association of socio-economic position across the life course and age at menopause: the British Women's Heart and Health Study. BJOG 2003; 110: 1078-1087.

18 Miller MR, Hankinson J, Brusasco V, et al. Standardisation of spirometry. Eur Respir J 2005; 26: 319-338.

19 Quanjer PH, Stanojevic S, Cole TJ, et al. Multi-ethnic reference values for spirometry for the 3-95-yr age range: the global lung function 2012 equations. Eur Respir J 2012; 40: 1324-1343.

20 Carlson CL, Cushman M, Enright PL, et al. Hormone replacement therapy is associated with higher FEV1 in elderly women. Am J Respir Crit Care Med 2001; 163: 423-428.

21 Pata $\mathrm{O}$, Atiş S, Utku Oz A, et al. The effects of hormone replacement therapy type on pulmonary functions in postmenopausal women. Maturitas 2003; 46: 213-218.

22 Cevrioglu AS, Fidan F, Unlu M, et al. The effects of hormone therapy on pulmonary function tests in postmenopausal women. Maturitas 2004; 49: 221-227.

23 Real FG, Svanes C, Macsali F, et al. Hormonal factors and respiratory health in women - a review. Clin Respir J 2008; 2: Suppl. 1, 111-119.

24 Rzymski P, Rzymski P, Tomczyk K, et al. Metal status in human endometrium: relation to cigarette smoking and histological lesions. Environ Res 2014; 132: 328-333.

25 Riggs BL, Khosla S, Melton LJ III. A unitary model for involutional osteoporosis: estrogen deficiency causes both type I and type II osteoporosis in postmenopausal women and contributes to bone loss in aging men. J Bone Miner Res 1998; 13: 763-773.

26 Leech JA, Dulberg C, Kellie S, et al. Relationship of lung function to severity of osteoporosis in women. Am Rev Respir Dis 1990; 141: 68-71.

27 Yang HL, Zhao L, Liu J, et al. Changes of pulmonary function for patients with osteoporotic vertebral compression fractures after kyphoplasty. J Spinal Disord Tech 2007; 20: 221-225.

28 Dong R, Chen L, Gu Y, et al. Improvement in respiratory function after vertebroplasty and kyphoplasty. Int Orthop 2009; 33: 1689-1694

29 Rode L, Bojesen SE, Weischer M, et al. Short telomere length, lung function and chronic obstructive pulmonary disease in 46,396 individuals. Thorax 2013; 68: 429-435.

30 Albrecht E, Sillanpää E, Karrasch S, et al. Telomere length in circulating leukocytes is associated with lung function and disease. Eur Respir J 2014; 43: 983-992.

31 Gray KE, Schiff MA, Fitzpatrick AL, et al. Leukocyte telomere length and age at menopause. Epidemiology 2014; 25: $139-146$.

32 Jacobsen BK, Heuch I, Kvåle G. Age at natural menopause and all-cause mortality: a 37-year follow-up of 19,731 Norwegian women. Am J Epidemiol 2003; 157: 923-929. 